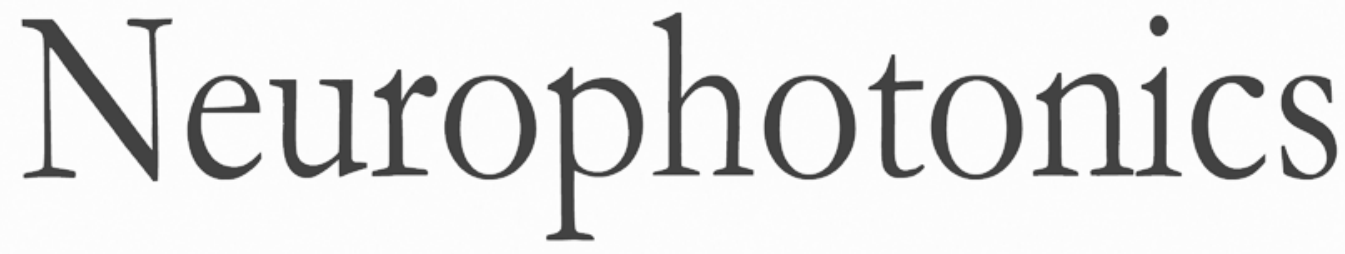

\title{
Imaging extracellular potassium dynamics in brain tissue using a potassium-sensitive nanosensor
}

Joel Wellbourne-Wood

Theresa S. Rimmele

Jean-Yves Chatton 


\title{
Imaging extracellular potassium dynamics in brain tissue using a potassium-sensitive nanosensor
}

\author{
Joel Wellbourne-Wood, ${ }^{a}$ Theresa S. Rimmele, ${ }^{a}$ and Jean-Yves Chatton ${ }^{\mathrm{a}, \mathrm{b}, \star}$ \\ aUniversity of Lausanne, Department of Fundamental Neurosciences, Lausanne, Switzerland \\ bUniversity of Lausanne, Cellular Imaging Facility, Lausanne, Switzerland
}

\begin{abstract}
Neuronal activity results in the release of $\mathrm{K}^{+}$into the extracellular space (ECS). Classically, measurements of extracellular $\mathrm{K}^{+}\left(\left[\mathrm{K}^{+}\right]_{0}\right)$ are carried out using $\mathrm{K}^{+}$-sensitive microelectrodes, which provide a single point measurement with undefined spatial resolution. An imaging approach would enable the spatiotemporal mapping of $\left[\mathrm{K}^{+}\right]_{0}$. Here, we report on the design and characterization of a fluorescence imaging-based $\mathrm{K}^{+}$-sensitive nanosensor for the ECS based on dendrimer nanotechnology. Spectral characterization, sensitivity, and selectivity of the nanosensor were assessed by spectrofluorimetry, as well as in both wide-field and two-photon microscopy settings, demonstrating the nanosensor efficacy over the physiologically relevant ion concentration range. Spatial and temporal kinetics of the nanosensor responses were assessed using a localized iontophoretic $\mathrm{K}^{+}$application on a two-photon imaging setup. Using acute mouse brain slices, we demonstrate that the nanosensor is retained in the ECS for extended periods of time. In addition, we present a ratiometric version of the nanosensor, validate its sensitivity in brain tissue in response to elicited neuronal activity and correlate the responses to the extracellular field potential. Together, this study demonstrates the efficacy of the $\mathrm{K}^{+}$-sensitive nanosensor approach and validates the possibility of creating multimodal nanosensors. $\odot$ The Authors. Published by SPIE under a Creative Commons Attribution 3.0 Unported License. Distribution or reproduction of this work in whole or in part requires full attribution of the original publication, including its DOI. [DOI: 10.1117/1.NPh.4.1.015002]
\end{abstract}

Keywords: extracellular space; potassium; potassium buffering; fluorescence microscopy; two-photon imaging; fluorescent indicator; nanotechnology.

Paper 16073RR received Dec. 9, 2016; accepted for publication Jan. 19, 2017; published online Feb. 9, 2017.

\section{Introduction}

During the repolarization phase of action potentials, neurons release potassium $\left(\mathrm{K}^{+}\right)$, causing an increase of the extracellular $\mathrm{K}^{+}$concentration $\left(\left[\mathrm{K}^{+}\right]_{\mathrm{o}}\right)$. Since $\mathrm{K}^{+}$is one of the key membrane potential determinants and, thus, fundamentally affects electrical signaling, the central nervous system has several homeostatic mechanisms to control $\left[\mathrm{K}^{+}\right]_{0}$. In addition to passive diffusion through the extracellular space $(\mathrm{ECS}), \mathrm{K}^{+}$is taken up and released by both glia and neurons, which is accomplished by various $\mathrm{K}^{+}$selective channels and transporters. ${ }^{1}$ In addition to its fundamental effects on neuronal excitability, changes in $\left[\mathrm{K}^{+}\right]_{\mathrm{o}}$ have been shown to influence long-term potentiation, ${ }^{2}$ vascular tone, ${ }^{3,4}$ and glial morphology. ${ }^{5}$ Impaired $\left[\mathrm{K}^{+}\right]_{\mathrm{o}}$ homeostasis is associated with a wide range of pathological states, such as epilepsy, ${ }^{6,7}$ cortical spreading depression, ${ }^{8}$ and ischemic stroke/ anoxia. ${ }^{9}$ It is yet unclear whether pathological changes in $\left[\mathrm{K}^{+}\right]_{\mathrm{o}}$ are a result or a cause of some of these diseases. To address the role of $\left[\mathrm{K}^{+}\right]_{\mathrm{o}}$ in physiological and in pathological conditions, monitoring the spatial and temporal dynamics of $\left[\mathrm{K}^{+}\right]_{\mathrm{o}}$ changes is of crucial importance.

To date, $\left[\mathrm{K}^{+}\right]_{\mathrm{o}}$ has been measured predominantly using $\mathrm{K}^{+}$-sensitive microelectrodes. ${ }^{10-12}$ Although still the methodological standard for measuring $\left[\mathrm{K}^{+}\right]_{\mathrm{o}}$, they are nonetheless limited by only providing a single point measurement. They also introduce a substantial dead space in the tissue, which may alter local structure and thus function. This makes their application limited in scope, especially from the perspective of monitoring

*Address all correspondence to: Jean-Yves Chatton, E-mail: jean-yves. chatton@unil.ch the spatiotemporal flux of $\mathrm{K}^{+}$, which is needed to address the standing questions regarding $\mathrm{K}^{+}$kinetics and dynamics.

Alternatively, one may attempt to image extracellular ion dynamics with ion sensitive dyes. This, however, is a far less widespread practice due to methodological problems, such as immediate diffusion of the dye through the ECS and a lack of dyes with a suitable affinity for their respective ion concentrations found in the ECS. Due to these methodological difficulties, published attempts to image $\mathrm{K}^{+}$in the ECS are limited to only a few studies. For instance, in one study, the authors utilized a long wavelength fluorescent sensor based on the tetraazachlorine moiety, which was applied to the exposed cortex of an anesthetized mouse to visualize $\mathrm{K}^{+}$waves in the cortex. ${ }^{13}$ Others injected the $\mathrm{K}^{+}$sensitive dye APG2 into the cisterna magna, and, after the diffusion of the dye throughout the brain, performed in vivo fluorescence imaging of the cortex. ${ }^{14}$ Both studies utilize in vivo fluorescence imaging strategies that, while highly valuable, only permit the imaging of superficial cortical regions.

To image various brain regions that are not accessible with current in vivo optical imaging methods, acute brain slice preparations can be used. This approach is also benefited by the ability to place microelectrodes more accurately and in the desired brain region for electrophysiology, as well as the ability to control the environment of the tissue to a greater degree. Imaging extracellular ion dynamics in acute slices is more difficult, however, due to the lack of an enclosed compartment that the skull and dura naturally provide, as well as the constant superfusion of artificial cerebrospinal fluid (ACSF) over the slice. Together, these cause a rapid washout of small molecule dyes from the ECS, with little to no time to gather useful data. 
To overcome these challenges, we designed a $\mathrm{K}^{+}$potassium nanosensor based on dendrimer nanotechnology. For this, we used a larger molecule [generation 5, polyethyleneglycol (PEG)functionalized, PAMAM dendrimer] as a scaffold for the small molecule $\mathrm{K}^{+}$sensitive dye Asante Potassium Green 4 (APG4) through a noncovalent interaction. This strategy of dye encapsulation has been previously applied in our laboratory for intracellular imaging with the sodium $\left(\mathrm{Na}^{+}\right)$sensitive dye, corona green. ${ }^{15}$ Here, we show that this new APG4-based nanosensor is well retained in the ECS, is selective and sensitive enough to measure $\left[\mathrm{K}^{+}\right]_{\mathrm{o}}$ at ionic concentration ranges relevant for ECS, and can be used to measure rapid local changes in $\left[\mathrm{K}^{+}\right]_{\mathrm{o}}$ in response to iontophoretic pulses of $\mathrm{K}^{+}$. Furthermore, we show changes in $\left[\mathrm{K}^{+}\right]_{\mathrm{o}}$ after the induction of neuronal activity with glutamate and correlate this to local field potential. Overall, we demonstrate that this APG4-based $\mathrm{K}^{+}$sensitive nanosensor is suitable for imaging $\left[\mathrm{K}^{+}\right]_{\mathrm{o}}$ in acute brain slices and can be used to monitor the spatiotemporal dynamics of $\left[\mathrm{K}^{+}\right]_{\mathrm{o}}$ in the brain.

\section{Materials and Methods}

\subsection{Nanosensor Synthesis}

Asante Potassium Green 4 (APG4), (Teflabs, Texas, USA) and Generation 5 PAMAM dendrimers with a 1,4-diaminobutane core and a poly (ethyleneglycol) methyl ether surface (Andrews ChemServices, Michigan) were used to prepare the nanosensor. The preparation of PAMAM dendrimer and APG4 was performed at a 1:1.2 molar ratio to ensure at least one dye molecule per dendrimer. For the ratiometric nanosensor, a second dye, AlexaFluor 568 (AF568) was incorporated, with a final 1.2:1.2:1 APG4 to AF568 to dendrimer ratio. To remove any unbound dye molecules after their integration into the dendrimer complex, the solution was passed through an ultrafiltration column with a $3 \mathrm{kDa}$ cutoff (Millipore, Massachusetts). Filtration was repeated several times to ensure a thorough washout of unbound dye. After being prepared, the nanosensor was stored at $-20^{\circ} \mathrm{C}$ in aqueous HEPES buffer at $\mathrm{pH}$ 7.5. Under these conditions, the nanosensor remains stable for $>1$ year.

\subsection{Spectral Characterizations}

Fluorimetric analysis was performed in quartz cuvettes using a using a spectrofluorimeter (LS55 Luminescence, PerkinElmer, Massachusetts). First, the nanosensor was diluted to $100 \mathrm{nM}$ in an extracellular-like solution containing $(\mathrm{mM}): \mathrm{NaCl} 160$, $\mathrm{KCl} / \mathrm{K}^{+}$-gluconate $0-50, \mathrm{CaCl}_{2} 1.3, \mathrm{MgSO}_{4} 0.81, \mathrm{NaH}_{2} \mathrm{PO}_{4}$ 0.78 , HEPES 20 adjusted to $\mathrm{pH} 7.4$ with $\mathrm{NaOH}$. For $\mathrm{K}^{+}$calibration curves, fluorescence intensities of the emission maxima were plotted against known $\mathrm{K}^{+}$concentrations. For this, K-gluconate $(1 \mathrm{M})$ was titrated in the cuvette over the range of 0 to $50 \mathrm{mM}$, and the results were corrected for dilution. For $\mathrm{Na}^{+}$ experiments, extracellular-like solution, as described above, was used but with $5 \mathrm{mM} \mathrm{KCl}$ and $135 \mathrm{mM} \mathrm{NaCl}$. NaCl (2M) was then titrated to gather data in the range of 135 to $165 \mathrm{mM} \mathrm{Na}^{+}$ and were also corrected for dilution. For $\mathrm{Mg}^{2+} \mathrm{Ca}^{2+}$, and $\mathrm{Ba}^{2+}$ experiments, $\mathrm{MgSO}_{4}, \mathrm{CaCl}_{2}$, and $\mathrm{Ba}(\mathrm{OH})_{2}$ (adjusted to $\mathrm{pH}$ 7.4) solutions were titrated in extracellular-like solution $\left(5 \mathrm{mM} \mathrm{K}^{+}\right)$, as described above. All spectral characterizations are an average of three separate scans.

\subsection{Sol-Gel Synthesis}

To immobilize the nanosensor for in vitro spatiotemporal imaging, silica sol-gels were synthesized in two steps: hydrolysis and condensation. ${ }^{16}$ The hydrolysis consists of the following reaction: $\mathrm{Si}(\mathrm{OMe})_{4}+\mathrm{MeOH}+\mathrm{H}_{2} \mathrm{O} \rightarrow \mathrm{MeOH}+\mathrm{Si}-\mathrm{OH}$, while the condensation follows the reaction: $\mathrm{Si}-\mathrm{OH}+\mathrm{Si}-\mathrm{OH} \rightarrow \mathrm{Si}-\mathrm{O}-\mathrm{Si}+$ $\mathrm{H}_{2} \mathrm{O}$. Hydrolysis was performed under a fume hood at room temperature by mixing $\mathrm{MeOH}, \mathrm{H}_{2} \mathrm{O}$, and $\mathrm{Si}(\mathrm{OMe})_{4}$ in molar ratios of 7.5:10:1. After hydrolysis, a small volume of fluorescent nanosensor preparation was diluted in $<100 \mu \mathrm{l}$ of the solgel mix and $5 \mu \mathrm{l}$ drops of the fluorescent sol-gel mix were pipetted onto glass microscope coverslips. Drops were allowed to condense and dry and were used for experiments after $24 \mathrm{~h}$.

\section{$2.4 K^{+}$lontophoresis}

Iontophoresis was carried out using a Neurophore BH-2 (Digitimer, Hertfordshire, United Kingdom). Borosilicate glass pipettes ( $\sim 5$ or $25 \mathrm{M} \Omega$ ) containing $1 \mathrm{M} \mathrm{KCl}$ were used. A DC holding current of $50 \mathrm{nA}$ was applied before and after iontophoretic pulses to ensure $\mathrm{K}^{+}$retention. $500 \mathrm{nA}$ pulses were used for rapid $\mathrm{K}^{+}$ejection.

\subsection{Imaging}

Single-photon fluorescence imaging of sol-gels was performed on an inverted epifluorescence microscope (Axiovert 100M, Carl Zeiss, Jena, Germany) using a $40 \times 1.3$ N.A. oil-immersion objective lens. Selection of the fluorescence excitation wavelength was performed using a monochromator (Till Photonics, Gräfelfing, Germany), and fluorescence was detected using an EM CCD camera (Andor, Belfast, United Kingdom). Image

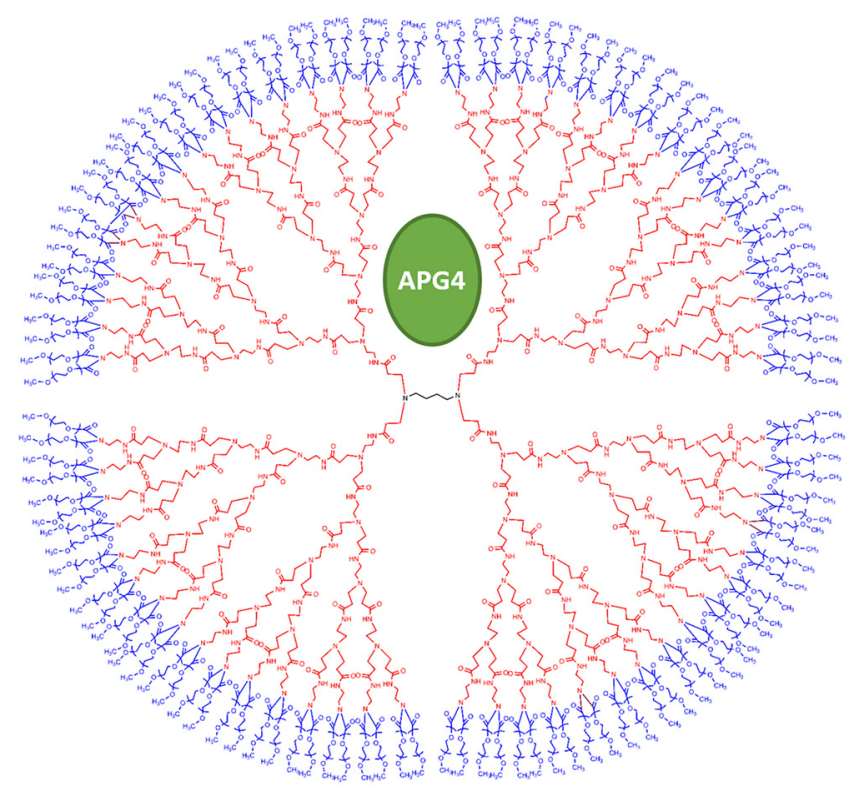

Fig. 1 Dendrimer-based potassium nanosensor. Schematic representation of the nanosensor design comprising a generation 5 (poly)amidoamine dendrimer (PAMAM; red) with a 1,4-diaminobutane core (DAB; black). The dendrimer is covalently functionalized with polyethyleneglycol dodecamers (PEG; blue) at its surface for biocompatibility and water solubility. The $\mathrm{K}^{+}$-sensitive indicator Asante Potassium Green 4 (APG4) is encapsulated in the dendrimer cavities at a molar ratio of $1: 1$ 
acquisition was computer-controlled using the Metafluor imaging software (Molecular Devices, California).

Two-photon imaging of sol-gels and acute brain slices was carried out using a custom-built two-photon microscope with a $40 \times 0.8$ N.A. water-dipping objective (Olympus). Fluorescence excitation was performed using a Chameleon Vision $\mathrm{S}$

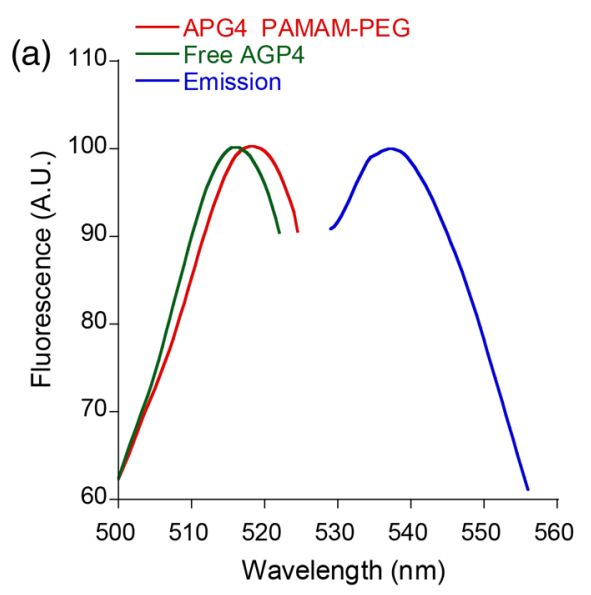

(c) $100 \rightarrow-$ APG4-PAMAM-PEG
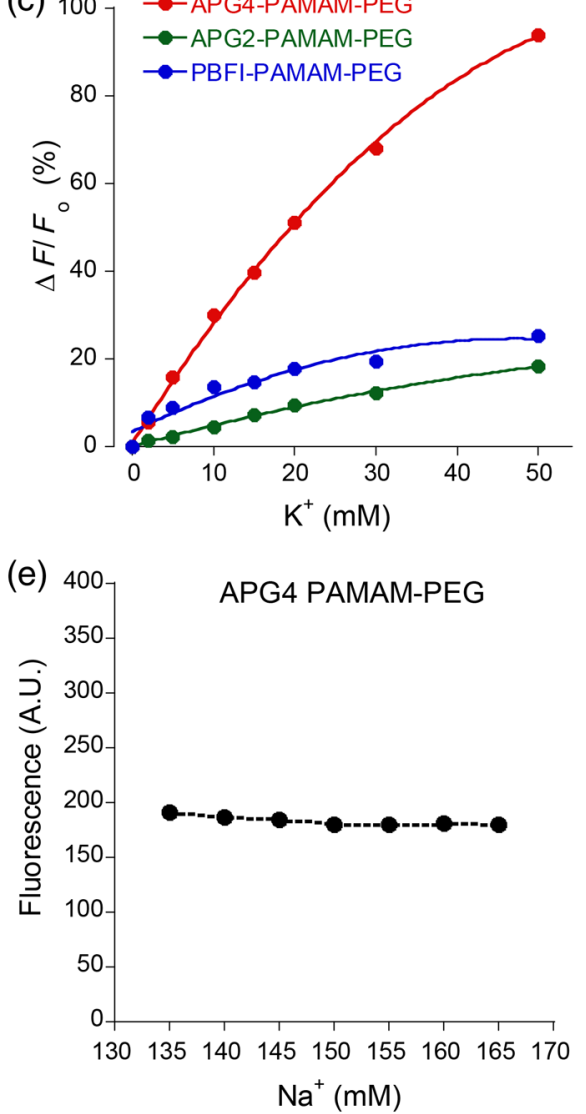

femtosecond infrared laser including group velocity dispersion compensation (Coherent, California). Captured emission wavelengths were $525 \pm 25 \mathrm{~nm}$ and $620 \pm 30 \mathrm{~nm}$ for the green and red channels, respectively. Image acquisition and linescan was performed using custom-written software in the Labview environment.
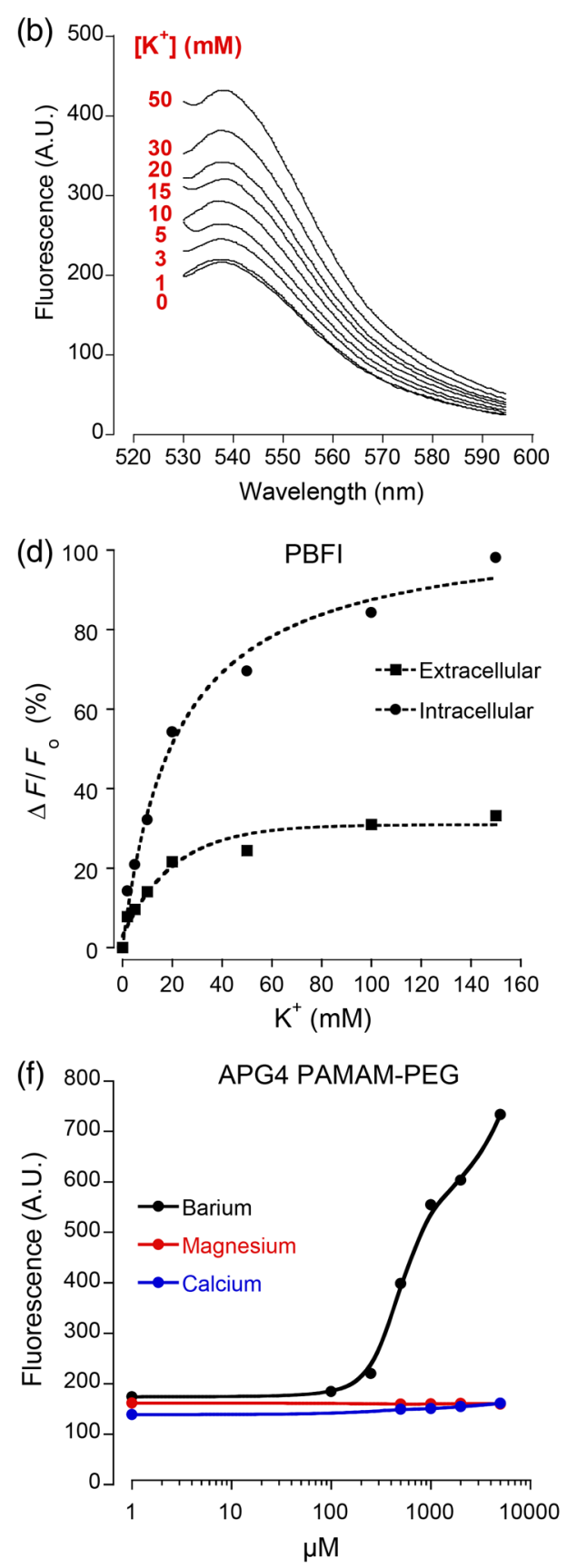

Fig. 2 Spectral properties and sensitivity of potassium nanosensors. (a) Single-photon excitation and emission spectra of the APG4 PAMAM-PEG nanosensor measured in a spectofluorimeter. (b) In vitro emission spectra $\left(\lambda_{\text {ex }} 515 \mathrm{~nm}\right)$ of APG4 PAMAM-PEG with a $\mathrm{K}^{+}$titration in the range 0 to $50 \mathrm{mM}$, corrected for dilution. (c) Comparison of sensitivity of three different $\mathrm{K}^{+}$-sensitive fluorescent indicators encapsulated in PAMAM dendrimers. The respective responsivity of APG4, APG2, and PBFI is shown for the same $\mathrm{K}^{+}$concentration range. (d) Differential sensitivities of the $\mathrm{K}^{+}$-indicator PBFI to $\mathrm{K}^{+}$ in solutions mimicking the extracellular or the intracellular milieu $\left(\lambda_{\mathrm{ex}} 330 \mathrm{~nm}\right)$ in response to $\mathrm{K}^{+}$titration ranging from 0 to $150 \mathrm{mM}$. Intracellular-like solutions contained $15 \mathrm{mM} \mathrm{Na}^{+}$, whereas extracellular-like solution contained $150 \mathrm{mM} \mathrm{Na}^{+}$. (e) APG4 PAMAM-PEG fluorescence in response to an increasing $\mathrm{Na}^{+}$ concentration spanning the extracellular physiological range, and ( $f$ ) to the divalent cations $\mathrm{Ca}^{2+}, \mathrm{Mg}^{2+}$, and $\mathrm{Ba}^{2+}$ measured at a fixed $5 \mathrm{mM} \mathrm{K}{ }^{+}$concentration emission $\left(\lambda_{\mathrm{ex}} 515 \mathrm{~nm}, \lambda_{\mathrm{em}} 537 \mathrm{~nm}\right)$. 


\subsection{Brain Slice Preparation}

After decapitation of 2.5- to 3-week old mice, brains were extracted and 300- $\mu \mathrm{m}$ thick hippocampal slices were prepared with a vibratome (VT 1000S, Leica, Wetzlar, Germany), as previously described. ${ }^{17}$ Slices were imaged in an open chamber superfused with ACSF, bubbled with $95 \% \mathrm{O}_{2} 5 \% \mathrm{CO}_{2}$. ACSF solution contains (mM): $\mathrm{NaCl} 125, \mathrm{KCl} 3, \mathrm{NaHCO}_{3} 25, \mathrm{CaCl}_{2}$ $1.2, \mathrm{MgSO}_{4} 1, \mathrm{NaH}_{2} \mathrm{PO}_{4} 1.25$, glucose 10 . Local delivery of the APG4 PAMAM-PEG nanosensor was achieved by applying pressure pulses of 5 to $60 \mathrm{~s}$ at 1 to 2 psi to the back of a glass pipette of tip diameter 1 to $2 \mu \mathrm{m}$ using a Pressure System II (The Toohey Company, Fairfield, New Jersey). Pipettes were retracted out of the tissue after satisfactory dye application.

All experimental procedures were carried out in strict accordance with the recommendations of the Swiss Ordinance on Animal Experimentation and were specifically approved for this study by the Veterinary Affairs Office of the Canton of Vaud, Switzerland.

\subsection{Extracellular Field Recordings}

Borosilicate glass pipettes $(\sim 4.5 \mathrm{M} \Omega)$ were filled with an ACSF-like solution. Recordings were obtained in the zero current clamp configuration using a Multiclamp 700B amplifier (Molecular Devices) in gap-free mode. Data were acquired at $10 \mathrm{kHz}$ by a Digidata 1440 analog-to-digital converter, controlled with the pCLAMP 10 software.

\section{Results}

\subsection{Nanosensor Design, Preparation, and Characterization}

The $\mathrm{K}^{+}$sensitive nanosensor is composed of a generation 5 poly (amidoamine) (PAMAM) type dendrimer with a poly(ethylenegylcol) functionalized surface. With our strategy, this G5 PAMAM-PEG dendrimer, which has been measured as $6.57 \mathrm{~nm}$ in diameter, ${ }^{15}$ becomes a host for small molecules, such as the $\mathrm{K}^{+}$sensitive dye APG4, via electrostatic interaction (Fig. 1). Tertiary amines that compose the dendrimer core are thought to play a key role in this incorporation process, since electrostatic interaction of any anionic dye with core amines will determine its integration pattern or lack thereof, as reported for other guest-host dendrimer interactions. ${ }^{18,19}$

A dye-to-dendrimer ratio of 1.2:1 was selected to ensure at least one dye per dendrimer molecule. After incorporation of the dye, the complex was repeatedly passed through a centrifugal filter with a 3-kDa cutoff to eliminate nonencapsulated dye. While each dendrimer most likely encapsulates a single APG4 molecule, the possibility that a single given dendrimer may contain fewer or more APG4 molecules has not been ruled out, as one has to consider the statistical nature of the nanoparticles produced.

Emission and excitation spectrum of the APG4 PAMAMPEG nanosensor in extracellular-like solution are presented in Fig. 2(a), along with the excitation spectrum of the free APG4 indicator. The spectra exhibit an excitation maximum around $515 \mathrm{~nm}$ and show a slightly red-shifted maximum excitation wavelength compared with free APG4. The emission maximum was found to be $\sim 540 \mathrm{~nm}$. The $\mathrm{K}^{+}$-dependent fluorescence was analyzed over a $\mathrm{K}^{+}$concentration range of 0 to $50 \mathrm{mM}$ [Fig. 2(b)] and showed that the $\mathrm{K}^{+}$-nanosensor fluorescence increases stepwise with increasing $\left[\mathrm{K}^{+}\right]$, and did not present spectral shifts in response to the increasing concentration. The peak emission intensity was plotted against $\left[\mathrm{K}^{+}\right]$and compared to two other nanosensors, APG2 PAMAM-PEG and PBFI PAMAM-PEG, which only differ in regards to their encapsulated fluorescent indicator [Fig. 2(c)]. The APG4-based nanosensor exhibits a fivefold higher fluorescent yield when compared to the APG2 and PBFI nanosensors in an extracellular-like milieu. It is noteworthy that the classical $\mathrm{K}^{+}$-indicator PBFI behaves drastically differently in solutions mimicking the intracellular and extracellular milieu [Fig. 2(d)], which may explain the poorer performance of PBFI PAMAM-PEG compared with the other two nanosensors.

Last, the sensitivity of the APG4 PAMAM-PEG nanosensor to $\mathrm{Na}^{+}$was assessed from 135 to $165 \mathrm{mM} \mathrm{Na}^{+}$, the concentration range expected to be found extracellularly under physiological conditions. Figure 2(e) indicates that altering $\left[\mathrm{Na}^{+}\right]$ has negligible effects on the overall nanosensor fluorescence while recorded with constant $\left[\mathrm{K}^{+}\right]$. Other cations were also tested for sensitivity, including magnesium, calcium, and barium [Fig. 2(f)]. While the APG4 PAMAM-PEG nanosensor is insensitive to calcium and magnesium over a relevant concentration range, the barium ion induces a large increase in fluorescence at concentrations greater than $100 \mu \mathrm{M}$. Since barium is known to block inwardly rectifying $\mathrm{K}^{+}$channels and is often used

(a)
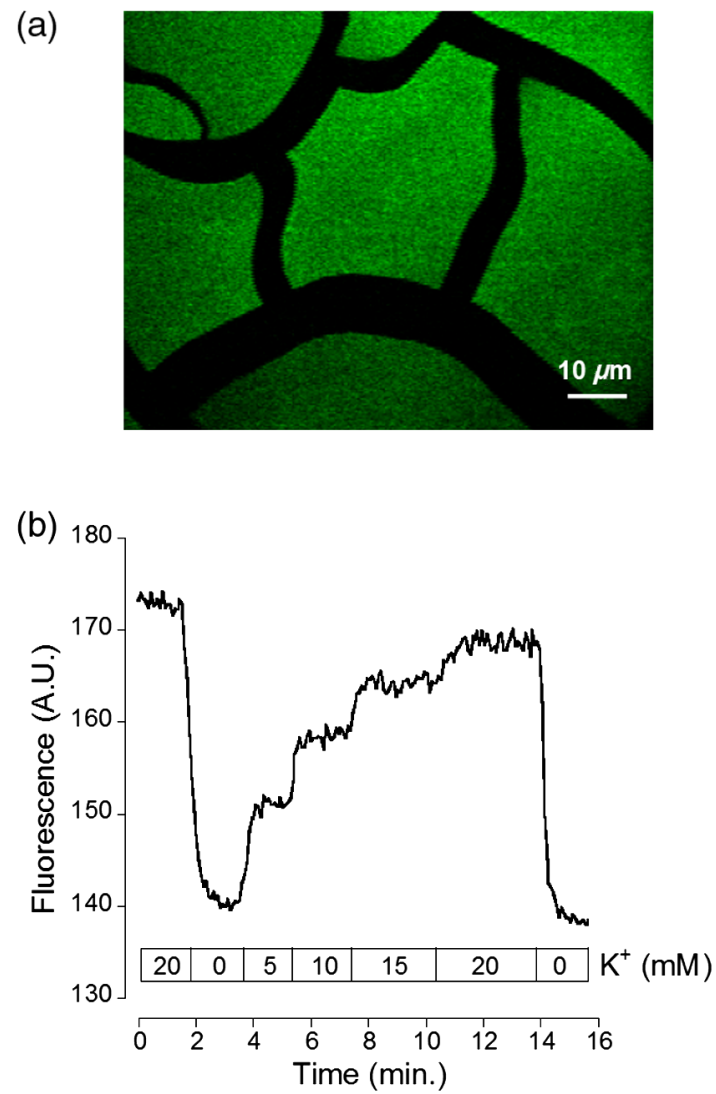

Fig. 3 Single-photon fluorescence imaging of APG4 PAMAM-PEG immobilized in a silica sol-gel. (a) Image of a condensed sol-gel preparation containing the APG4 PAMAM-PEG nanosensor. During the process of condensation, the thin layer of silica sol-gel attached to the coverslip cracks into small fragments. (b) Fluorescence recording of the nanosensor embedded in sol-gels during superfusion of extracellular-like solutions containing $0,5,10,15$, or $20 \mathrm{mM} \mathrm{K}^{+}$, and showing the responsiveness and reversibility of sensor response. 
experimentally, ${ }^{20}$ caution must be employed when using the nanosensor.

After demonstrating the sensitivity of the nanoprobe in a cuvette, we then investigated its spatiotemporal characteristics using single and two-photon microscopy in vitro. For this purpose, we incorporated the nanosensor into a porous silica glass using a sol-gel process, providing us with an in vitro platform in which we could image its performance, all the while keeping it immobilized, as well as closely controlling the local environment via iontophoresis or superfusion of solutions.

After preparation of the sol-gel silica structures on cover slips [Fig. 3(a)], we performed single-photon imaging in a closed perfusion chamber, in which we could measure the stepwise increase in fluorescence following increasing concentrations of $\mathrm{K}^{+}$. Superfusion and imaging of the sol-gel fragments demonstrated that its porosity is sufficient for allowing fast diffusion of solutes through their matrix, while being able to immobilize the nanosensor. The stepwise increase in fluorescence and the reversibility of the signal upon washout of the high $\mathrm{K}^{+}$solution demonstrate that the dye is stable and responsive over time [Fig. 3(b)].
Because two-photon microscopy is significantly more advantageous for imaging in thick tissue and it is primarily the setting in which this nanosensor is ultimately intended to be employed, we analyzed the APG4 PAMAM-PEG containing silica glass in a two-photon microscope. This allowed us to verify its efficacy in a two-photon excitation setting, as well as to gather additional data regarding spatial and temporal kinetics of the sensor. To demonstrate the excitation maxima in a two-photon setting, wavelength tuning from 770 to $840 \mathrm{~nm}$ was performed. The emission intensity was recorded for each wavelength, correcting for laser output power as measured in the beam path. Figure 4(a) shows the measured two-photon excitation spectrum, showing a maximal peak two-photon excitation wavelength for the nanosensor at $\sim 815 \mathrm{~nm}$.

To analyze the spatial and temporal kinetics of the sensor, iontophoretic $\mathrm{K}^{+}$ejection was performed. A $1 \mathrm{M} \mathrm{KCl}$ filled borosilicate glass pipette was lowered to a distance of $5 \mu \mathrm{m}$ from the surface of the sol-gel, and iontophoretic pulses were used to expel $\mathrm{K}^{+}$, thereby increasing the local $\mathrm{K}^{+}$concentration in a radial manner from the pipette tip [Fig. 4(b)]. A local fluorescence increase that was maximal at the tip of the pipette opening and radiating outward could be observed. Figure 4(c) shows

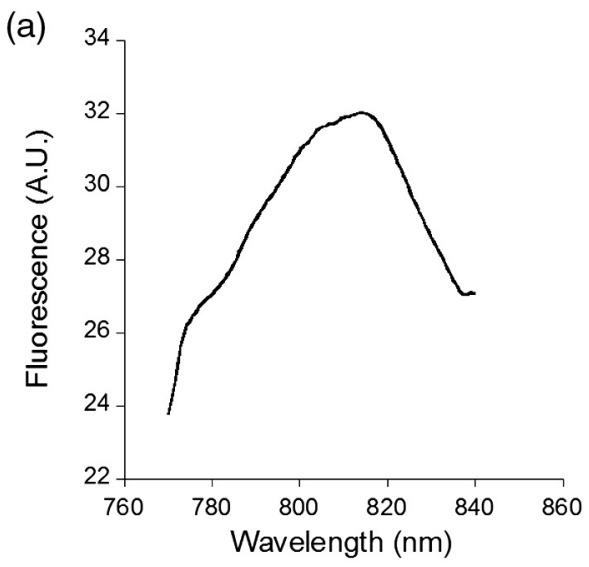

(b)

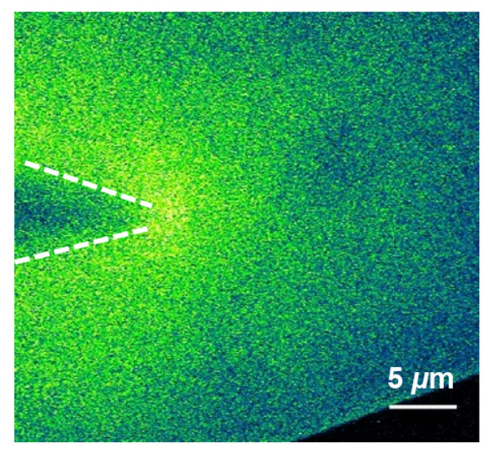

(c)

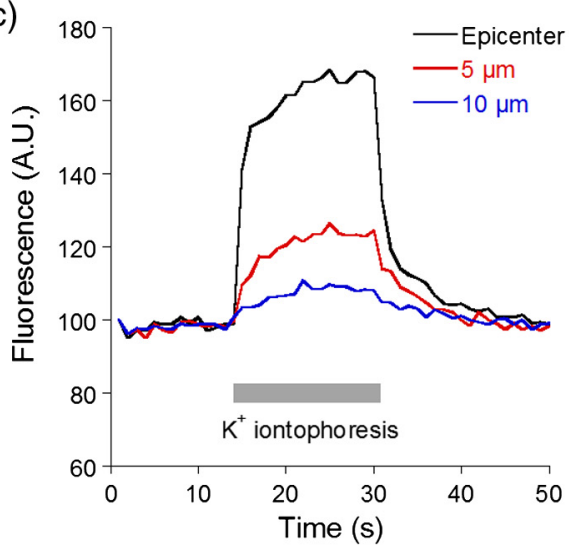

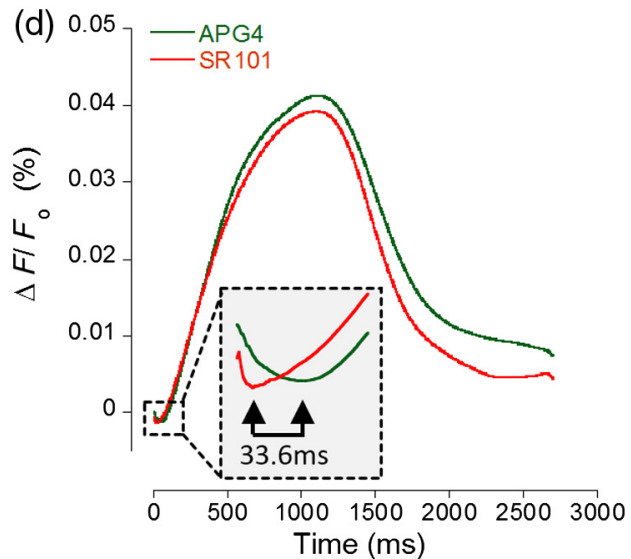

Fig. 4 Two-photon imaging of APG4 PAMAM-PEG immobilized in a silica sol-gel. (a) Two-photon excitation spectra of APG4 PAMAM-PEG measured over the range 770 to $840 \mathrm{~nm}$. (b) Local fluorescence increase of nanosensor containing sol-gel emanating from the focal point of an iontophoretic pulse. The dotted white line depicts the pipette positioned at the interface of the sol-gel. (c) Kinetics of sol-gel fluorescence responding to the release of $\mathrm{K}^{+}$via iontophoretic pulses measured at regions 0,5 , and $10 \mu \mathrm{m}$ from the epicenter. Measurements were done at a sampling rate of $1 \mathrm{~Hz}$. (d) Fast kinetics (sampling rate: $1000 \mathrm{~Hz}$ ) line scanning of sol-gel fluorescence in response to an iontophoretic pulse of both $\mathrm{K}^{+}$and the fluorescent dye SR101. The green channel represents the fluorescence intensity change of the $\mathrm{K}^{+}$nanosensor embedded inside the silica sol-gel in response to the fast delivery of $\mathrm{K}^{+}$, while the red channel is an indicator of the rate of cation extrusion from the pipette and used to estimate the response delay of the nanosensor response to abrupt $\mathrm{K}^{+}$changes, estimated to be $33.6 \mathrm{~ms}$. 
the waveform of fluorescence at regions of varying distance from the epicenter, demonstrating spatial variation of the $\mathrm{K}^{+}$ concentration increase and the resulting fluorescence. The characteristically slow increase to steady-state and fast decay of signal also matches the kinetics of iontophoretic release, as demonstrated previously by others. ${ }^{21}$ Taken together, these results indicate that the temporal and spatial characteristics of fluorescence of the nanosensor are favorable and that the signal is an accurate representation of the $\mathrm{K}^{+}$fluxes induced. To further explore the temporal kinetics of the nanosensor, line-scanning experiments were performed during iontophoresis. The pipette was placed on the surface of the sol-gel, and simultaneous iontophoresis of sulforhodamine 101 (SR101) and $\mathrm{K}^{+}$was performed, causing a measurable increase in fluorescence of both the red channel (SR101 expelled from the pipette) and the green channel [APG4 PAMAM-PEG embedded in the sol-gel; Fig. 4(d)]. The presence of the two fluorescent dyes in the pipette allowed us to take into account the nonlinear kinetics of iontophoresis. ${ }^{21}$ The inflection points of the fluorescence signals were compared as an indication of rapid kinetics of the sensor. The slope of the fluorescence of the red channel reaches its inflection point $33.6 \mathrm{~ms}$ before the slope of the green channel. This delay is a good estimate of the responsiveness of the nanosensor in the presence of a sudden $\mathrm{K}^{+}$rise. By comparison, a fast version of the genetically encoded calcium indicator, GCaMP6F, has an activation time of $74 \mathrm{~ms}^{22}$ Synthetic organic ion indicators are generally faster. A range of hypothetical calcium dyes with different $K_{\mathrm{d}}$ values binding $\mathrm{Ca}^{2+}$ over a range of concentrations will have a response time between 2 to $40 \mathrm{~ms},{ }^{23}$ while the widely used dye fura- 2 demonstrates a response time estimated at $11 \mathrm{~ms}$ in-vitro and $40 \mathrm{~ms}$ in-vivo. ${ }^{24,25}$

\subsection{Acute Brain Slice Measurements}

The second issue related to imaging $\mathrm{K}^{+}$in the ECS is the retention of the dye in the tissue. Injecting a small water-soluble molecule such as APG4 into acute brain slices results in a fast disappearance of the dye that makes it impossible to use effectively. Therefore, the dendrimer must serve the function of stabilizing the dye in the ECS for extended periods of time. Figure 5(a) displays the nanosensor's distribution in the ECS after several microinjections demonstrating concurrent fluorescence of the nanosensor (green), staining of astrocytes with SR101 (red), and unlabeled cell bodies likely belonging to neurons. A comparison between the injections of free APG4 and APG4 PAMAM-PEG demonstrates that the dendrimer greatly improves the nanosensor retention in tissue and that the dendrimer is successfully being retained in the ECS [Fig. 5(b)].

The marked difference in dye signal over time demonstrates the efficacy of the nanosensor design. Occasionally, observed decay of signal after injection in the tissue was found to be mainly due to bleaching, with minimal contribution of washout of the dye, demonstrating that the dye is very well stabilized by the dendrimer in the ECS (data not shown).

To demonstrate sensitivity of the nanosensor in tissue, iontophoresis of $\mathrm{K}^{+}$in acute slices was carried out with or without the presence of 4-aminopyridine (4-AP, $50 \mu \mathrm{M}$ ) in the perfusion solution (Fig. 6). The amplitude of signal increase was higher for slices perfused with 4-AP. This observation is consistent with the fact that 4-AP is known to block a number of $\mathrm{K}^{+}$ channels $^{26}$ and may, therefore, alter $\mathrm{K}^{+}$buffering out of the ECS. This likely slows the process of $\mathrm{K}^{+}$uptake by cells in response to the extracellular concentration increase via iontophoresis. (a)

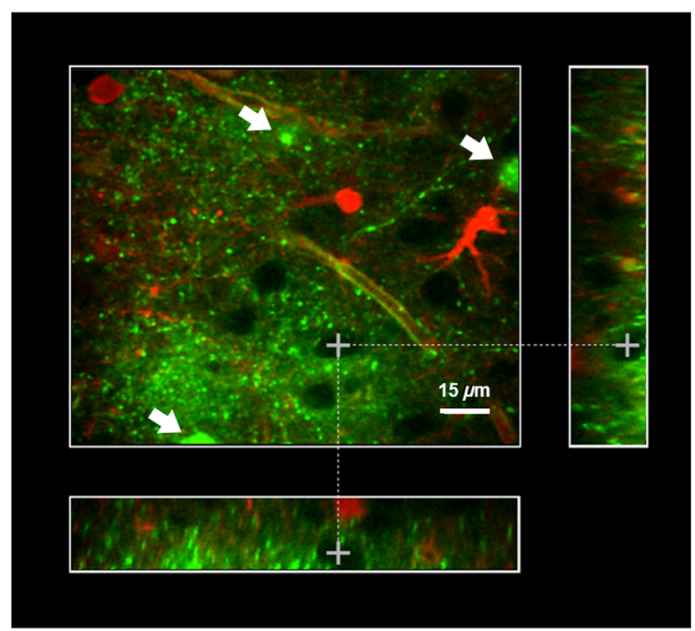

(b)

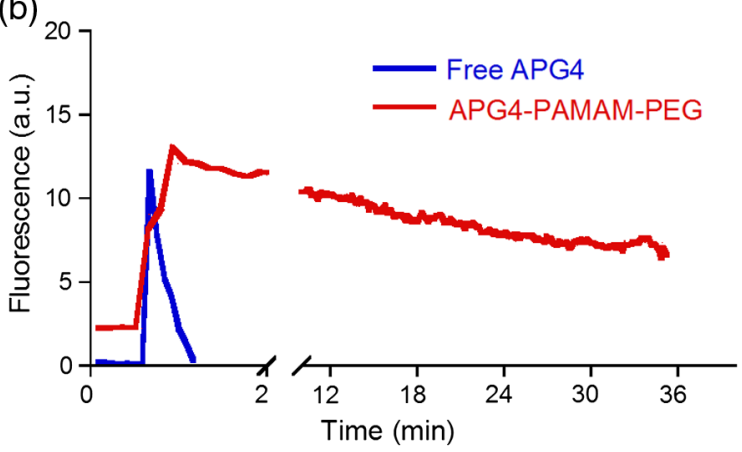

Fig. 5 Retention of the potassium nanosensor in the ECS. (a) Fluorescence of the nanosensor (green) injected in the ECS as measured by two-photon microscopy in a mouse acute brain slice $\left(\lambda_{\text {ex }} 800 \mathrm{~nm}\right)$ with concurrent SR101 staining of glial cells (red). White arrows indicate microinjection sites. (b) Fluorescence retention of APG4 of free APG4 locally applied compared with the retention of the APG4 PAMAM-PEG nanosensor in the same conditions.

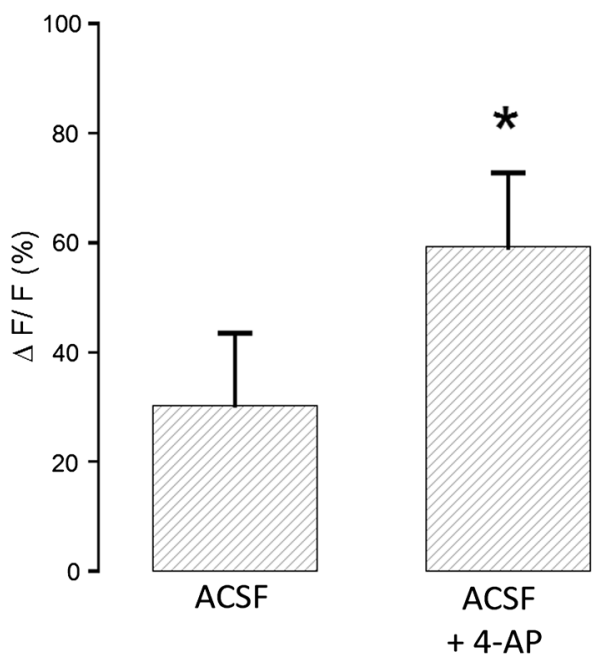

Fig. $6 \mathrm{~K}^{+}$iontophoresis in acute brain slices. Measurement of fluorescence of the APG4 PAMAM-PEG nanosensor after iontophoresis of $\mathrm{K}^{+}$in acute brain slices. Measurements were performed in the absence and in the presence of the $\mathrm{K}^{+}$channel blocker 4-AP $(50 \mu \mathrm{M}, n=3)$. 
One challenge related to imaging brain tissue in a two-photon setting is that it is very sensitive to small movements or drift, which greatly affects the thin focal plane. This is especially true in regards to imaging the ECS. While individual cells show relatively stable signal even with small focal plane shifts, the fine and heterogeneous structures of the ECS are, in our experience, extremely prone to such shifts. Such movement artifacts cannot be avoided entirely, especially with long duration recordings. To counteract this problem, we designed another version of the APG4-based nanosensor. This improved version includes a second, potassium insensitive dye, AlexaFluor 568 (AF568), and is thus dubbed the APG4/AF568 PAMAM-PEG nanosensor [Fig. 7(a)]. Its emission spectral scans [Fig. 7(b)] demonstrate that the sensitivity of the APG4 is not diminished by the addition of another molecule in the dendrimer. The AF568 does not respond to potassium and emits in the red spectrum. This allows for more precise ratiometric imaging of $\mathrm{K}^{+}$dynamics and demonstrates the general feasibility of the ratiometric, multidye, and multimodal nanosensor design.

(a)
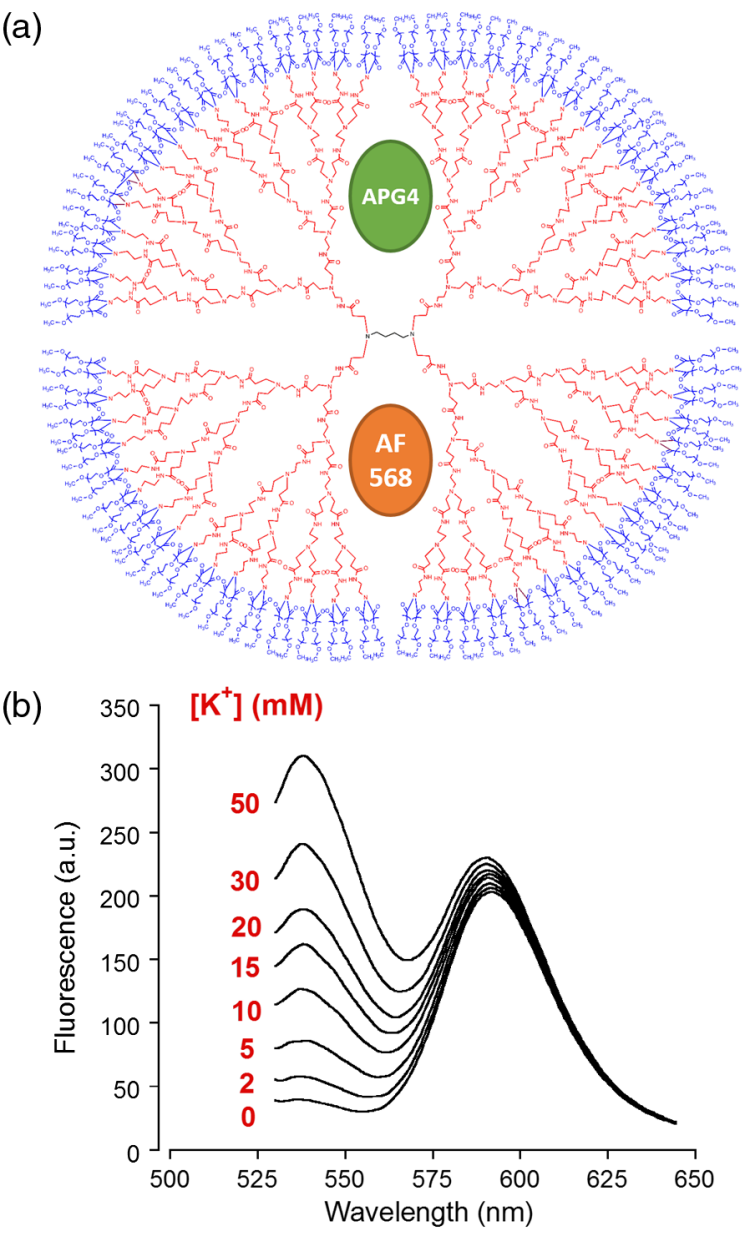

Fig. 7 Spectral properties and potassium sensitivity of the APG4/ AF568 PAMAM-PEG ratiometric nanosensor. (a) Schematic representation of the nanosensor design comprising a generation 5 (poly)amidoamine dendrimer (PAMAM; red) with a 1,4-diaminobutane core (DAB; black), as well a polyethyleneglycol (PEG; blue) outer shell, as depicted in Fig. 1. This dual-dye ratiometric version encapsulates the $\mathrm{K}^{+}$-sensitive indicator Asante Potassium Green 4 (APG4) and the $\mathrm{K}^{+}$-insensitive dye Alexa Fluor 568 (AF568) in a 1:1 molar ratio. (b) Single-photon in vitro emission spectra $\left(\lambda_{\mathrm{ex}} 515 \mathrm{~nm}\right)$ of the ratiometric nanosensor with a $\mathrm{K}^{+}$titration in the range $0-50 \mathrm{mM}$, corrected for dilution.

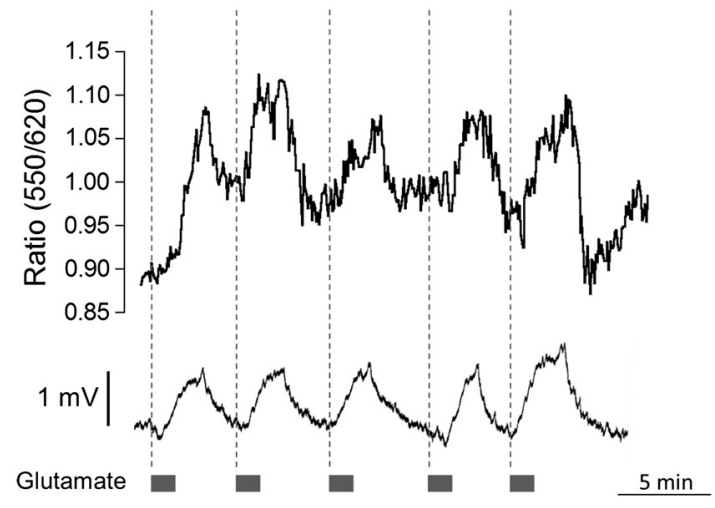

Fig. 8 Two-photon imaging of APG4/AF568 PAMAM-PEG in acute brain slices. Ratio of green over red fluorescence detection channel of the potassium nanosensor in a $5 \mu \mathrm{m}$ diameter region (top panel, $\lambda_{\mathrm{ex}}$ $800 \mathrm{~nm}$ ) and local field potential ( $\mathrm{mV}$, bottom panel) in response to repetitive bath applications of $500-\mu \mathrm{M}$ glutamate.

While the basal level of $\left[\mathrm{K}^{+}\right]_{\mathrm{o}}$ in the mammal brain rests around 2.5 to $3 \mathrm{mM}$, periods of intense neuronal activity can raise that value to a physiological ceiling level of around 10 to $12 \mathrm{mM} .{ }^{27}$ It was also shown recently with $\mathrm{K}^{+}$-sensitive microelectrodes that bath application of $500-\mu \mathrm{M}$ glutamate induces a rise of $\left[\mathrm{K}^{+}\right]_{\mathrm{o}}$ in the range of several millimolar in the "stratum radiatum" of the CA1. ${ }^{28}$ Using the APG4/AF568 PAMAM-PEG nanosensor, we performed ratiometric imaging of $\mathrm{K}^{+}$in the stratum radiatum of the CA1 in response to intermittent bath application of $500-\mu \mathrm{M}$ glutamate (Fig. 8). In this experiment, spatial resolution was privileged by selecting one small, $5-\mu \mathrm{m}$ diameter region of interest. The signal-to-noise ratio of the trace would increase for larger regions. An application time of $30 \mathrm{~s}$ was chosen since $\left[\mathrm{K}^{+}\right]_{\mathrm{o}}$ elevations typically follow sustained and persistent neural activity.

With each sequential application, the ratio of fluorescence of the two channels rises and then drops accordingly. The corresponding field potential was simultaneously recorded, demonstrating a similar waveform. Individual peaks exhibiting common characteristics in both the field potential and fluorescence can be observed, further supporting the efficacy of the nanosensor design.

\section{Discussion}

During neuronal activity, ion fluxes across cell membranes are associated both with the generation of action potentials and with the postsynaptic events. $\left[\mathrm{K}^{+}\right]_{0}$ levels have been shown to undergo substantial fluctuations in brain tissue during physiological and pathological conditions. In particular, neuronal excitation leads to elevations of $\left[\mathrm{K}^{+}\right]_{\mathrm{o}}$ that have to be rapidly regulated to enable subsequent activity. ${ }^{29}$ Once locally released in the ECS by neurons, $\left[\mathrm{K}^{+}\right]_{\mathrm{o}}$ elevations are rapidly dissipated by passive diffusion across the ECS, reuptake into neurons and astrocytes, and redistribution across the astroglial syncytium. ${ }^{1}$

The measurement of $\left[\mathrm{K}^{+}\right]_{\mathrm{o}}$ has been so far mainly performed using ion-selective electrodes. ${ }^{30,31}$ These approaches are relatively invasive and do not provide direct spatial information about $\mathrm{K}^{+}$changes. Fluorescence-based detection of ECS $\mathrm{K}^{+}$ offers the benefits of being both noninvasive and capable of assessing the spatiotemporal pattern of $\mathrm{K}^{+}$flux within the tissue. The advantages of spatiotemporal data acquisition in regards to ion dynamics are evident when one considers the wealth 
of knowledge uncovered, for instance, in the field of $\mathrm{Ca}^{2+}$ signaling.

The ECS in the brain occupies $15 \%$ to $30 \%$ of the volume, and its geometry is made of an interconnected network of $<100 \mathrm{nM}$ tube- or sheet-like conduits. ${ }^{32}$ Due to the limited volume and diffusion constraints of the ECS, the local neuronal $\mathrm{K}^{+}$ efflux (e.g., occurring during the repolarization phase of action potentials) is sufficient to significantly increase $\left[\mathrm{K}^{+}\right]_{0}$. While the basal $\left[\mathrm{K}^{+}\right]_{\mathrm{o}}$ concentration is $\sim 3 \mathrm{mM},{ }^{33}$ somewhat lower than the plasma concentration, $\left[\mathrm{K}^{+}\right]_{\mathrm{o}}$ may rise to 10 to $12 \mathrm{mM}$ during sustained neuronal activity. ${ }^{27}$ While the ECS presents some diffusion barriers due to its constrained geometry, diffusion of small molecules in the ECS is relatively fast. In particular, organic fluorescent molecules routinely used for ion imaging have molecular weights typically $<1500 \mathrm{Da}$. Local application of such dyes in the ECS leads to disappearance of fluorescent signal within seconds, as seen in Fig. 5(b). The strategy that we followed was to incorporate a $\mathrm{K}^{+}$-selective indicator inside a larger scaffold consisting of a generation 5 PAMAM dendrimer. The size of this construct, estimated to be around $6.6 \mathrm{~nm}$ in diameter with a molecular mass above $100 \mathrm{kDa},{ }^{15}$ considerably slows down its diffusion in the tissue and enables longer term monitoring of ECS fluorescent signals. It should be underlined that smaller dendrimers, i.e., under generation 4 , do not form developed enough internal cavities to provide encapsulation, while dendrimers of generation 7 and above are too large, resulting in blocked pores that are less accessible. ${ }^{34}$ Generation 5 provides internal cavities large enough to host smaller molecules, such as APG4, and still open and exposed for solutes and ions to readily pass through.

Another important factor is surface functionalization. To prevent toxicity of the dendrimer-based nanosensor, the surface of the dendrimer must be electroneutral. It has been demonstrated that naked PAMAM dendrimers, which possess cationic amine terminals, induce the formation of transient holes in membranes of living cells. ${ }^{35}$ This increases toxicity and allows passage to solutes, including the dendrimer itself, across the plasma membrane. In the same study, a PEG functionalized-dendrimer was shown to have no effect over the same concentration range. It has also been shown that PEG-functionalized dendrimers, when inserted inside neurons, do not affect the integrity or electrical properties of the neurons. ${ }^{15}$

While the dendrimer nanoparticle properties play an important role in the nanosensor design, the dye itself is especially important. Due to the fact that $\mathrm{Na}^{+}$and $\mathrm{K}^{+}$are both monovalent cations with very similar properties, dyes that bind $\mathrm{K}^{+}$are likely to have a certain degree of affinity to $\mathrm{Na}^{+}$. Most ion-sensitive dyes are used to image "intracellular" ion dynamics, but due to differential ionic concentrations across the membrane, some dyes that are effective intracellularly will not be as useful in the extracellular setting. The classical $\mathrm{K}^{+}$dye $\mathrm{PBFI},{ }^{36}$ for example, is more effective intracellularly, where there is a low concentration of $\mathrm{Na}^{+}$and a high concentration of $\mathrm{K}^{+}$. Extracellularly, the respective ion concentrations are reversed, and PBFI loses sensitivity due to the confounding fluorescence introduced by the high $\mathrm{Na}^{+}$concentration [Fig. 2(d)]. For this reason, it was important to assess the sensitivity of nanosensors based on various dyes in an extracellular-like solution. Figure 2(c) demonstrates that under extracellular conditions, APG4 is the most sensitive of the dyes tested.

The APG4 fluorescent nanosensor emits green light at $\sim 540 \mathrm{nM}$, with a one-photon excitation maximum at $\sim 490 \mathrm{~nm}$.
In two-photon mode, it has a broader excitation centered around $810 \mathrm{~nm}$. As various organic dyes with red emission have similar two-photon excitation wavelengths around $800 \mathrm{~nm}$, one could envisage using one common excitation wavelength while detecting both green and red emission simultaneously. This indeed proved useful for our design, enabling the improvement of the nanosensor via the incorporation of AF568, which can be excited with the same infrared wavelength.

After characterizing the nanosensor using sol-gels and cuvettes, we aimed to demonstrate its efficacy in living brain tissue. Iontophoresis in the tissue or superfusion of solutions are both possible methods for demonstrating differential fluorescence of the nanosensor in the tissue in response to a variable concentration of $\left[\mathrm{K}^{+}\right]_{0}$. However, it is more relevant to both physiology and pathology to assess whether the nanosensor is able to measure changes associated with $\left[\mathrm{K}^{+}\right]_{\mathrm{o}}$, where the source of the $\mathrm{K}^{+}$fluctuations is the tissue itself.

The experiment carried out in Fig. 8 demonstrates that the sensor is sensitive enough to detect such changes in $\left[\mathrm{K}^{+}\right]_{\mathrm{o}}$ and correlates them to a well-established readout, the local field potential. To relate raw fluorescence to concentrations, one would need a calibration protocol for the nanosensor in living tissue. This would require the silencing of neuronal activity, as well as the blockade of a variety of $\mathrm{K}^{+}$channels, using molecules such as 4-AP. Barium can also be used to block $\mathrm{K}^{+}$channels, but the observed sensitivity of the nanosensor to barium may exclude it from employment in the calibration process, except at low concentrations $(<100 \mu \mathrm{M})$. Alternatively, one could envisage extending the sol-gel approach depicted in Figs. 3 and 4 and combining it with the ratiometric nanosensor to calibrate the fluorescence signal ex vivo. This approach, once validated, would significantly accelerate the process of obtaining absolute extracellular $\mathrm{K}^{+}$levels in situ.

$\mathrm{K}^{+}$homeostasis has many contributing functional components taking part in the process, including Na-K-ATPase pumps, Na-K-Cl cotransporters (NKCC), inwardly rectifying potassium channels $\left(\mathrm{K}_{\mathrm{ir}}\right)$, voltage-gated potassium channels, and, in the case of the astrocytic syncytium, gap junctions. ${ }^{1}$ This nanosensor is likely to facilitate future investigations of these mechanisms and their interactions. The spatiotemporal nature of the gathered data will allow for the investigation into the possible existence of $\mathrm{K}^{+}$microdomains around activated neurons and the spatial extent of these domains.

In addition to validating the feasibility of the nanosensor for imaging in the ECS, this study highlights the range of possible extensions of the nanosensor strategy. For example, the ratiometric nanosensor design demonstrates the possibility of combining several optical readouts, laying the path for the design of multimodal optical nanosensors and introducing a broader range of possible applications.

\section{Disclosures}

The authors declare no financial or personal conflicts of interest.

\section{Acknowledgments}

This work was supported by Grant \# 31003a_159513 from the Swiss National Science Foundation to J.-Y. Chatton. We thank Enea Pianezzi for his help during the first phases of the study. We also thank Egbert Welker, who very generously donated the BH2 Neurophore iontophoresis unit. 


\section{References}

1. P. Kofuji and E. A. Newman, "Potassium buffering in the central nervous system," Neuroscience 129(4), 1043-1054 (2004).

2. B. A. Ballyk and J. W. Goh, "Elevation of extracellular potassium facilitates the induction of hippocampal long-term potentiation," J. Neurosci. Res. 33(4), 598-604 (1992).

3. W. Kuschinsky et al., "Perivascular potassium and $\mathrm{pH}$ as determinants of local pial arterial diameter in cats. A microapplication study," Circ. Res. 31(2), 240-247 (1972).

4. J. McCulloch, L. Edvinsson, and P. Watt, "Comparison of the effects of potassium and $\mathrm{pH}$ on the calibre of cerebral veins and arteries," Pflugers Arch. 393(1), 95-98 (1982).

5. K. S. Canady, F. Ali-Osman, and E. W. Rubel, "Extracellular potassium influences DNA and protein syntheses and glial fibrillary acidic protein expression in cultured glial cells," Glia 3(5), 368-374 (1990).

6. D. K. Binder et al., "Increased seizure duration and slowed potassium kinetics in mice lacking aquaporin-4 water channels," Glia 53(6), 631636 (2006).

7. U. Heinemann et al., "Alterations of glial cell function in temporal lobe epilepsy," Epilepsia 41(Suppl 6), S185-S189 (2000).

8. R. Enger et al., "Dynamics of ionic shifts in cortical spreading depression," Cereb. Cortex 25(11), 4469-4476 (2015).

9. T. J. Sick, Z. C. Feng, and M. Rosenthal, "Spatial stability of extracellular potassium ion and blood flow distribution in rat cerebral cortex after permanent middle cerebral artery occlusion," J. Cereb. Blood Flow Metab. 18(10), 1114-1120 (1998).

10. S. Adamek and F. Vyskocil, "Potassium-selective microelectrode revealed difference in threshold potassium concentration for cortical spreading depression in female and male rat brain," Brain Res. 1370, 215-219 (2011).

11. A. J. Hansen, H. Lund-Andersen, and C. Crone, " $\mathrm{K}^{+}$-permeability of the blood-brain barrier, investigated by aid of a $\mathrm{K}^{+}$-sensitive microelectrode," Acta Physiol. Scand. 101(4), 438-445 (1977).

12. F. Vyskocil, N. Kritz, and J. Bures, "Potassium-selective microelectrodes used for measuring the extracellular brain potassium during spreading depression and anoxic depolarization in rats," Brain Res. 39(1), 255-259 (1972).

13. P. Padmawar et al., " $\mathrm{K}^{+}$waves in brain cortex visualized using a longwavelength $\mathrm{K}^{+}$-sensing fluorescent indicator," Nat. Methods 2(11), 825-827 (2005).

14. P. Bazzigaluppi, S. Dufour, and P. L. Carlen, "Wide field fluorescent imaging of extracellular spatiotemporal potassium dynamics in vivo," Neuroimage 104, 110-116 (2015).

15. C. M. Lamy et al., "Sodium sensing in neurons with a dendrimer-based nanoprobe," ACS Nano 6(2), 1176-1187 (2012).

16. C. J. Brinker, "Hydrolysis and condensation of silicates-effects on structure," J. Non-Cryst. Solids 100(1-3), 31-50 (1988).

17. B. Tahvildari et al., "Selective functional interactions between excitatory and inhibitory cortical neurons and differential contribution to persistent activity of the slow oscillation," J. Neurosci. 32(35), 1216512179 (2012).

18. M. W. Baars et al., "The localization of guests in water-soluble oligoethyleneoxy-modified poly(propylene imine) dendrimers," Angew. Chem. Int. Ed. Engl. 39(7), 1285-1288 (2000).

19. F. Wang, N. Shao, and Y. Cheng, "Paramagnetic NMR investigation of dendrimer-based host-guest interactions," PLoS One 8(6), e64722 (2013).

20. C. M. Armstrong and S. R. Taylor, "Interaction of barium ions with potassium channels in squid giant axons," Biophys. J. 30(3), 473488 (1980).

21. R. D. Purves, "The physics of iontophoretic pipettes," J. Neurosci. Methods 1(2), 165-178 (1979).
22. B. Podor et al., "Comparison of genetically encoded calcium indicators for monitoring action potentials in mammalian brain by two-photon excitation fluorescence microscopy," Neurophotonics 2(2), 021014 (2015).

23. M. B. Cannell and S. H. Cody, "Fluorescent ion indicators," in Handbook of Biological Confocal Microscopy, J. B. Pawley, Ed., 3rd ed., pp. 741-742, Springer Science, New York (2006).

24. A. P. Jackson et al., "The kinetics of calcium binding to fura-2 and indo1," FEBS Lett. 216(1), 35-39 (1987).

25. S. M. Baylor and S. Hollingworth, "Fura-2 calcium transients in frog skeletal muscle fibres," J. Physiol. 403, 151-192 (1988).

26. B. Rudy, "Diversity and ubiquity of K channels," Neuroscience 25(3), 729-749 (1988).

27. U. Heinemann and H. D. Lux, "Ceiling of stimulus induced rises in extracellular potassium concentration in the cerebral cortex of cat," Brain Res. 120(2), 231-249 (1977).

28. N. Haack et al., "Double-barreled and concentric microelectrodes for measurement of extracellular ion signals in brain tissue," J. Visualized Exp. 103, 53058 (2015).

29. T. Amedee, A. Robert, and J. A. Coles, "Potassium homeostasis and glial energy metabolism," Glia 21, 46-55 (1997).

30. S. Dufour et al., "In vivo simultaneous intra- and extracellular potassium recordings using a micro-optrode," J. Neurosci. Methods 194(2), 206217 (2011).

31. U. Heinemann and H. D. Lux, "Undershoots following stimulusinduced rises of extracellular potassium concentration in cerebral cortex of cat," Brain Res. 93(1), 63-76 (1975).

32. A. D. Wong et al., "The blood-brain barrier: an engineering perspective," Front. Neuroeng. 6, 7 (2013).

33. R. Katzman, "Maintenance of a constant brain extracellular potassium," Fed. Proc. 35(6), 1244-1247 (1976).

34. A. R. Menjoge, R. M. Kannan, and D. A. Tomalia, "Dendrimer-based drug and imaging conjugates: design considerations for nanomedical applications," Drug Discov. Today 15(5-6), 171-185 (2010).

35. S. Hong et al., "Interaction of polycationic polymers with supported lipid bilayers and cells: nanoscale hole formation and enhanced membrane permeability," Bioconjugate Chem. 17(3), 728-734 (2006).

36. A. Minta and R. Y. Tsien, "Fluorescent indicators for cytosolic sodium," J. Biol. Chem. 264(32), 19449-19457 (1989).

Joel Wellbourne-Wood obtained his MSc at the University of Regensburg, Germany. He is now working toward his PhD at the University of Lausanne, Switzerland, where he is studying the role of potassium dynamics in physiology and pathology.

Theresa S. Rimmele received her undergraduate degree in biology from University of Tuebingen, Germany, and her PhD in life sciences from University of Lausanne, Switzerland, studying the interaction between glial cells and neurons. Her research focus was on the influence of extracellular potassium on the dynamics of astrocytic glutamate uptake. In her postgraduate studies at Boston Children's Hospital, she investigates the function of the glutamate transporter GLT-1 in neurons.

Jean-Yves Chatton received his PhD degree in pharmacology from the University of Lausanne, Switzerland, followed by postdocs at NIH (Bethesda, Maryland) and Bern (Switzerland). He now leads a research lab in neuroscience and is the head of the Cellular Imaging Facility at University of Lausanne. His research interests include the understanding of neuron-glia interactions, mainly related to bioenergetics and ion homeostasis, in health and disease. He is active in developing and employing imaging and fluorescence technologies to investigate these issues. 